\title{
LOS CONFLICTOS EN LOS DESTINOS TURÍSTICOS DURANTE LA PANDEMIA DE LA COVID-19 DESDE LA PERSPECTIVA DE LOS MOVIMIENTOS SOCIALES. El caso de Málaga
}

Conflicts in tourist destinations during the COVID-19 pandemic from social movements perspective. The case study of Málaga

\author{
Fátima Santos-Izquierdo Bueno \\ Universidad de Málaga \\ fatimasantos@uma.es \\ https://orcid.org/0000-0002-3122-773X \\ Marina Montiel Cesares \\ Universidad de Málaga \\ marina98mc@uma.es \\ https://orcid.org/0000-0002-8544-5505

\section{Yolanda Romero Padilla} \\ Universidad Complutense de Madrid \\ yromerop@ucm.es \\ https://orcid.org/0000-0001-9753-5113
}

RESUMEN: Los espacios urbanos han experimentado un considerable crecimiento turístico en las últimas décadas, especialmente tras la crisis económica de 2008, momento a partir del cual comenzaron a intensificarse las voces críticas. Los movimientos sociales de resistencia denunciaban los efectos negativos del modelo turístico imperante. Ahora, la crisis provocada por la Covid-19 ha contribuido a visibilizar la fragilidad y conflictos presentes en estos destinos. El estudio pretende responder a la pregunta de si la Covid-19 ha supuesto un cambio en la percepción de los movimientos sociales sobre los conflictos en los espacios turísticos. Se utiliza para ello un enfoque cualitativo aplicado a movimientos sociales del ámbito de estudio de Málaga. Los resultados de la revisión teórica son coincidentes con lo observado en el caso de estudio. Existe una dicotomía en cuanto a la percepción de cómo será el escenario post-Covid basada en dos elementos. Por un lado, la continuidad e incluso intensificación que están experimentando las dinámicas del modelo de 
crecimiento turístico-inmobiliario presentes en el contexto pre-pandemia. Por otro, las oportunidades para transformar el sector turístico dada la fragilidad mostrada durante la crisis sanitaria, así como por la progresiva concienciación social sobre la emergencia climática.

Palabras clave: turismo, conflictos, movimientos sociales, Covid-19.

RESUM: Els espais urbans han experimentat un considerable creixement turístic en les últimes dècades, especialment després de la crisi econòmica de 2008 , moment a partir del qual van començar a intensificar-se les veus crítiques. Els moviments socials de resistència denunciaven els efectes negatius del model turístic imperant. Ara, la crisi provocada per la Covid-19 ha contribuït a visibilitzar la fragilitat i conflictes presents en aquests destins. L'estudi pretén respondre a la pregunta de si la Covid-19 ha suposat un canvi en la percepció dels moviments socials sobre els conflictes en els espais turístics. S'utilitza per a això un enfocament qualitatiu aplicat a moviments socials de l'àmbit d'estudi de Màlaga. Els resultats de la revisió teòrica són coincidents amb l'observat en el cas d'estudi. Existeix una dicotomia quant a la percepció de com serà l'escenari post-Covid basada en dos elements. D'una banda, la continuïtat $\mathrm{i}$ fins $\mathrm{i}$ tot intensificació que estan experimentant les dinàmiques del model de creixement turístic-immobiliari presents en el context pre-pandèmia. Per un altre, les oportunitats per a transformar el sector turístic donada la fragilitat mostrada durant la crisi sanitària, així com per la progressiva conscienciació social sobre l'emergència climàtica.

Paraules Clau: turisme, conflictes, moviments socials, Covid-19.

ABSTRACT: Urban spaces have experienced a considerable tourist growth in recent decades, mostly after the 2008 economic crisis, a time from which critical voices began to intensify. Social resistance movements denounced the negative effects of the prevailing tourism model. Now, the Covid-19 recession has contributed to making visible the fragility and conflicts present in these destinations. The study aims to answer the question of whether Covid-19 has brought about a change in perception of social movements regarding conflicts in tourist destinations. It uses a qualitative approach applied to social move- 
ments in the Malaga study area. The results of the theoretical review coincide with it was observed in the case study. Related to perceptions, there is a dichotomy about how will be the post-Covid scenario based on two elements. On the one hand, the continuity and even intensification of the tourism-real estate growth model dynamics present in the pre-pandemic context. On the other hand, the tourism sector transformation opportunities given the fragility shown during the health crisis, as well as by the progressive social awareness of the climate emergency.

KEYWORDS: tourism, conflicts, social movements, Covid-19

\section{Introducción}

$\mathrm{L}$ a crisis sanitaria provocada por la Covid-19 ha puesto en jaque al mundo y a los fundamentos de funcionamiento de la economía neoliberal. Un año y medio después de que saltasen las alarmas en China, las cifras superan los 130 millones de personas contagiadas en todo el mundo y la estimación de fallecidos se acerca a los 3 millones (Ministerio de Sanidad 2021), si bien se estima que las cifras pueden ser mucho mayores por la carencia o errores de registro en muchos países. Esta crisis sanitaria supone un fuerte cambio de hábitos en la población (confinamiento domiciliario o territorial, toque de queda, cierre de fronteras, etc.) y la restricción sobre actividades no esenciales en muchos países. Todo ello ha propiciado que la crisis sanitaria se extienda también hacia una crisis socioeconómica.

Para el turismo, la aparición de la Covid-19 ha supuesto la paralización y caída de los viajes internacionales, cuya recuperación sigue envuelta en un escenario de incertidumbre. Las restricciones a la movilidad y a las actividades no esenciales afectan directamente a la esencia de la actividad turística, basada fundamentalmente en desplazamientos y en el ocio. Según los datos del Barómetro OMT del Turismo Mundial (UNWTO 2021), en 2020 las llegadas de turistas internacionales sufrieron una caída del 74\% respecto al año anterior y una disminución estimada de 1,3 billones de dólares en ingresos de exportaciones. Los países con mayor peso del sector turístico en el conjunto de su economía son los que mayor impacto están experimentando desde el punto de vista económico y social, especialmente por su efecto en el empleo. 
En España, de acuerdo con los datos del Instituto Nacional de Estadística (Hosteltur, 2021) en 2020 el número de llegadas de turistas internacionales descendió un 77,3\% y los ingresos un 78,5\% respecto al año anterior. Por su parte, los datos del sindicato Comisiones Obreras, durante el año 2020, muestran cómo medio millón de personas trabajadoras en el sector turístico podrían haber perdido su empleo, encontrándose, hasta el mes de noviembre, 340.372 personas en ERTE, hecho que no aseguraba su futura reincorporación, ya que a partir de diciembre comenzaron a acumularse despidos de personas en esta situación (Murray \& Cañada 2021).

Este escenario de crisis llega en un momento en el que muchos espacios turísticos europeos mostraban síntomas de conflictos ocasionados por los desajustes del crecimiento turístico reciente en las ciudades (OMT 2018). Ejemplo de ello son las investigaciones que identifican y analizan situaciones conflictivas en torno a proyectos y procesos de crecimiento urbano-turístico a través de la aparición de movimientos sociales (Milano, 2018; Navarro-Jurado et al. 2019). Partiendo de estas corrientes de investigación, este trabajo se plantea la cuestión de si la Covid-19 ha supuesto un cambio en la percepción de los movimientos sociales sobre los conflictos en los espacios turísticos. La investigación se desarrolla bajo la premisa de que esta crisis sanitaria supone una oportunidad para la reflexión sobre cómo va a ser el escenario post-Covid. En este sentido, desde las empresas e instituciones se han propuesto medidas cortoplacistas y coyunturales ligadas a paliar los efectos socioeconómicos de la crisis, pero también se encuentran otras a medio y largo plazo que podrían dar paso a cambios estructurales en el modelo de desarrollo previo a la pandemia. Prueba de esto último es la vinculación del Plan de Recuperación para Europa con el Pacto Verde Europeo (Parlamento Europeo 2020), lo cual enmarca la recuperación de la crisis de la Covid-19 en el contexto de emergencia climática. En España esta misma reflexión se aplica además sobre el modelo turístico y las posibilidades de su evolución futura: los que reclaman la vuelta a la normalidad turística anterior sin atender a sus desajustes y conflictos y los que apuestan por cambiar el antiguo modelo turístico de crecimiento ilimitado (Romero-Padilla et al. 2020).

Por tanto, el objetivo de este artículo es explorar desde un punto de vista cualitativo la percepción que los movimientos sociales tienen de los efectos 
de la crisis sanitaria sobre los conflictos en espacios turísticos. Para ello se realiza una revisión conceptual de las aportaciones que se han realizado en la literatura académica sobre los conflictos en espacios turísticos en un contexto pre-Covid-19 así como las aportaciones que se han realizado sobre la crisis sanitaria desde el ámbito turístico. Posteriormente se presentan los detalles metodológicos del estudio cualitativo y el ámbito de estudio (Málaga). Finalmente se analizan y discuten los resultados del estudio y se concluye con futuras líneas de investigación.

\section{Estado de la cuestión}

\section{I Contexto pre-Covid-19}

Los orígenes del modelo de turismo urbano prepandémico pueden situarse en la reestructuración económica posfordista norteamericana, cuando las ciudades se orientaron a los servicios, en particular, a los sectores FIRE (en inglés: finance, insurance y real state) y se sentaron las bases económicas de las ciudades globales que dieron forma al nuevo régimen urbano de acumulación de capital (Soja 2008). Este modelo postindustrial comienza a implantarse en las ciudades españolas consolidadas a partir de los 70, a través de procesos planificados de regeneración y rehabilitación urbana para impulsar la revalorización e inversión en dichas zonas (Jover \& Díaz-Parra 2020, Russo \& Scarnato 2018). Esfuerzos que consiguieron incrementar el atractivo residencial, pero también el turístico (De la Calle 2019).

Existen dos tipos de intereses que determinan el futuro de las ciudades: las fuerzas que presionan por el "valor de cambio" y aquellas que lo hacen por el "valor de uso" (Lefebvre 1974). En la nueva ciudad posfordista, las fuerzas que persiguen el valor de cambio pueden configurar estrategias locales a través de una coalición de agentes políticos y económicos que persigue el crecimiento urbano con el objetivo de incrementar el uso de la tierra y maximizar las rentas urbanas, en detrimento de aquellos que luchan por el valor de uso (Harvey 2013, Logan \& Molotch 1987). En este sentido, en el contexto pre-Covid pudimos presenciar cómo la industria turística se configuró como una de las principales y más dinámicas fuerzas productoras del espacio en el sentido de Lefebvre (1974), durante el denominado "giro recreativo" 
de la ciudad postindustrial que ha ido convirtiendo las ciudades en lugares de consumo, espectáculo y turismo (Bugalski 2020, García-Hernández et al. 2020, Ioannides et al. 2019, Nofre et al. 2018). Lo que Blanco-Romero et al. (2019) denominaron la mercantilización de la ciudad multifuncional en la era de la restructuración turística de la ciudad.

Este proceso de turistificación planetaria se aceleró tras la crisis financiera de 2008, cuando el crecimiento turístico se constituyó como uno de los principales modelos de acumulación y reproducción del capital para la recuperación de la crisis (Cañada et al. 2021, Fletcher et al. 2019). Además, el crecimiento turístico fue legitimado, mediante el discurso político de la generación de empleo y economías de escala, como un "agente vinculante social" asumiéndose su círculo virtuoso de creación de riqueza, lo que reforzaba su papel como motor de la máquina de crecimiento urbano, siguiendo el concepto empleado Logan y Molotch (1987). En este contexto, se aplicaron toda una serie de medidas liberalizadoras por parte de los gobiernos (por ejemplo, la Directiva Bolkestein 2009) que, en convergencia con otros factores (las compañías aéreas low-cost, proliferación de ofertas de alojamiento y transporte mediante el capitalismo de plataforma...), resultaron en facilidades para la implantación de las actividades turísticas y el abaratamiento del precio del viaje y la estancia (Capocchi et al. 2019, De la Calle 2019 Oklevik et al. 2019).

Todo esto produjo un notable aumento en la afluencia de visitantes en gran parte de los destinos europeos más populares: Ámsterdam, Atenas, Barcelona, Dubrovnik, Lisboa, Málaga, Palma de Mallorca, etc. (Fletcher et al. 2019, Milano 2018). Motivó la aparición de respuestas críticas, protestas vecinales y movimientos sociales de resistencia; así como la introducción en el debate público y académico de nuevos términos como "overtourism" o turismofobia (Blanco-Romero et al. 2019, Navarro-Jurado et al. 2019). El turismo se integra así con un papel relevante en la geografía urbana de los conflictos (Del Romero, 2018).

En este contexto, aparecen estudios que muestran la existencia de dos formas diferentes de entender la ciudad turística por parte de actores cuyas expectativas y deseos entran en colisión como es el valor de uso para la vida cotidiana frente al valor de cambio para el rendimiento económico o 
el crecimiento turístico ilimitado frente a un modelo de turismo responsable (Blanco-Romero et al. 2019, Milano 2018; Hiernaux \& González 2014, Navarro-Jurado et al. 2019). Romero-Padilla et al. (2019) sistematizan esta dicotomía a través del análisis de conflictos en espacios turísticos, comparando las características de la perspectiva crecentista y las reivindicaciones de los movimientos sociales involucrados en el conflicto:

Tabla 1. Comparación de características: crecimiento y exceso del turismo y las demandas de los movimientos sociales.

\begin{tabular}{|c|c|c|}
\hline Características & Crecimiento & $\begin{array}{l}\text { Reivindicaciones movimientos } \\
\text { sociales }\end{array}$ \\
\hline $\begin{array}{l}\text { Dimensión } \\
\text { territorial- } \\
\text { urbana- } \\
\text { ambiental }\end{array}$ & $\begin{array}{l}\text { Manda lo Global } \\
\text { Legislación y Derecho líquidos } \\
\text { Desigualdad urbana, injusticia } \\
\text { ambiental y espacial } \\
\text { Vivienda mercantilizada (fondos } \\
\text { de inversión - turistas) } \\
\text { Espacio público privado } \\
\text { (terrazas, eventos marketing...) }\end{array}$ & $\begin{array}{l}\text { Manda lo Local } \\
\text { Legislación y Derecho sólidos } \\
\text { Derecho a la ciudad, justicia } \\
\text { ambiental y espacial } \\
\text { Vivienda para vivir (propiedad } \\
\text { individual y alquiler) } \\
\text { Espacio público común (vida } \\
\text { comunitaria, reuniones, manifes- } \\
\text { taciones...) }\end{array}$ \\
\hline $\begin{array}{l}\text { Dimensión } \\
\text { social y } \\
\text { subjetividad } \\
\text { colectiva }\end{array}$ & $\begin{array}{l}\text { Tiempos de transformaciones } \\
\text { rápidas } \\
\text { Democracia representativa o } \\
\text { delegativa } \\
\text { Nutrición turística, vida mercan- } \\
\text { tilizada } \\
\text { Identidad genérica global }\end{array}$ & $\begin{array}{l}\text { Tiempos de transformaciones } \\
\text { lentas } \\
\text { Democracia directa } \\
\text { Forma de vida cotidiana, común } \\
\text { Identidad colectiva propia, local }\end{array}$ \\
\hline $\begin{array}{l}\text { Dimensión } \\
\text { turística- } \\
\text { económica }\end{array}$ & $\begin{array}{l}\text { Economía basada en la compe- } \\
\text { tencia entre ciudades } \\
\text { Manda el valor de cambio } \\
\text { Manda el Negocio } \\
\text { Protagonismo del capital finan- } \\
\text { ciero e inversores externos } \\
\text { Turismo de alta densidad y } \\
\text { genérico }\end{array}$ & $\begin{array}{l}\text { Economía local y redes de coo- } \\
\text { peración } \\
\text { Manda el valor de uso } \\
\text { Manda el Ocio } \\
\text { Protagonismo del pequeño } \\
\text { comercio de barrio y de los } \\
\text { agentes locales } \\
\text { Turismo de baja densidad y res- } \\
\text { ponsable }\end{array}$ \\
\hline
\end{tabular}

Fuente: Romero-Padilla et al. (2019).

Un elemento a destacar en este escenario fue que, incluso desde organismos internacionales como la OMT (2018) se reconocieron los impactos del excesivo crecimiento turístico en los destinos, en la calidad de vida de sus residentes y en la propia calidad de la experiencia de los turistas. Sin embargo, 
estos organismos propusieron como solución la mejora en la gestión estratégica del turismo a partir de un conjunto de técnicas, algunas conducentes a la elitización de los destinos (Trancoso 2018) que conseguían sortear el problema sin abordarlo de forma estructural (Milano 2018).

En el lado opuesto, nos encontramos con una serie de reflexiones y análisis académicos desde enfoques críticos que interpretan el discurso y aparición de los movimientos sociales de resistencia como una reivindicación por el derecho a la ciudad y el "valor de uso" en el sentido de Lefebvre (1968). La existencia de resistencias se plantea como cuestionamiento del imperativo de crecimiento y su capacidad para generar e intensificar las desigualdades económicas y extra-económicas (radicales, de género, étnicas) así como la destrucción de los entornos urbanos y naturales (Logan \& Molotch 1987, Soja 2008). En este contexto, comienzan a surgir corrientes que plantean otros escenarios, como el de la prosperidad sin crecimiento (Jackson 2009) o el potencial de transformación del decrecimiento, que intentan poner límites a las ansias de reproducción del capital (Fletcher et al. 2019, Navarro-Jurado et al. 2019; Perkumienė \& Pranskūnienè 2019). La llegada de la Covid-19, como se muestra a continuación, ha reforzado el interés por estas corrientes y las posibilidades de un cambio de paradigma impulsado por la necesidad de atender la crisis sanitaria de manera vinculada a la emergencia climática.

\subsection{Contexto Covid-19}

El 11 de marzo de 2020, la Covid-19 es declarada pandemia por la Organización Mundial de la Salud (OMS ); días más tarde, la declaración del Estado de Alarma en España supuso la paralización de la actividad turística, junto con el resto de las actividades económicas no esenciales. No obstante, el sector turístico ha sido uno de los más perjudicados debido a que la actividad turística, y especialmente el modelo de desarrollo turístico dominante, depende de la movilidad y las interacciones humanas (Cañada \& Murray 2021).

En el nuevo contexto, los destinos con una economía extremadamente dependiente de los ingresos del turismo — máxime los que dependen del turismo internacional - se han convertido en los más frágiles, ya que han tenido que enfrentarse al descenso de la demanda turística, la clausura de 
flujos aéreos de acceso a los destinos y el cierre de empresas y negocios locales, con todos los impactos socioeconómicos derivados (Aledo et al. 2021). Un sector turístico que ya venía arrastrando, desde la crisis económica de 2008, la mayor desprotección sobre el empleo y los salarios más bajos (Mansilla 2021), y que podría utilizar esta crisis para intensificar esta y otras dinámicas (Alexandri \& Janoschka 2020).

Asimismo, en este nuevo contexto, entidades públicas y privadas del sector presentaron escenarios-propuesta desde enfoques cortoplacistas y economicistas que buscaban el business as usual prepandémico (por ejemplo: OMT, Exceltur o Bain y Ernst\&Young) (Romero-Padilla et al. 2020). En lo que parecía un intento por poner en marcha el conocido como manual del capitalismo del desastre (Klein 2007, Fletcher et al. 2021), dejando de lado los requisitos en materia de sostenibilidad y cambio climático (Gössling et al. 2020, Ioannides \& Gyimóthy 2020).

Pese a esto, se observa un cambio de visión a lo largo del desarrollo de la pandemia vinculado con la progresiva toma de conciencia de que la crisis sanitaria no podía abordarse en el corto plazo. De esta forma, desde organismos internacionales se comenzaron a plantear reflexiones que parecían insertar la estrategia de recuperación de la crisis en el actual contexto de emergencia climática, desde instituciones como la ONU (2020) o la Unión Europea a través de la presentación de unos fondos de recuperación económica basados en los principios del Pacto Verde Europeo, donde se explicitaba el objetivo de construir una Europa más ecológica y resiliente (Comisión Europea 2020). Siguiendo esta línea, la ONU (2020) publicó una hoja de ruta para la recuperación titulada "La Covid-19 y la transformación del turismo" donde destacó la importancia de "crear comunidades y empresas más resilientes mediante la innovación, la digitalización, la sostenibilidad y las alianzas" (ONU 2020:2). Con base en la investigación de Romero et al. (2020), se realiza una comparativa entre aquellas medidas cortoplacistas con diagnósticos unidimensionales (económicos) y las medidas a largo plazo que incluyen las tres dimensiones de la sostenibilidad (ambiental, sociocultural y medioambiental): 
Tabla II. Comparación entre medidas economicistas a corto plazo y medidas sostenibles a medio-largo plazo.

\begin{tabular}{|l|l|}
\hline $\begin{array}{l}\text { Corto plazo } \\
\text { con diagnóstico unidimensional }\end{array}$ & $\begin{array}{l}\text { Largo plazo } \\
\text { con diagnóstico multidimensional }\end{array}$ \\
\hline Situación actual: diagnósticos inestables. & Objetivos de Desarrollo Sostenible de \\
Visión económica. & Naciones Unidas y Pacto Verde de la Unión \\
Causas coyunturales. & Europea. \\
Reproducir mismo modelo & Causas estructurales. \\
pre-Covid-19. & Cambiar: nuevo modelo \\
& post-Covid-19. \\
\hline
\end{tabular}

Fuente: Elaboración propia con base en Romero-Padilla et al. (2020).

En cuanto a algunas dinámicas destacables desarrolladas en el presente contexto pandémico, nos encontramos con la insistencia del mercado de los apartamentos turísticos por continuar especulando con las rentas del suelo (Cocola-Gant 2021) así como con la ausencia de normas que incluyan medidas para atajar las causas de la dependencia turístico-inmobiliaria (Valdivielso \& Adrover 2021). Pese al progresivo cambio de visión del sector hacia el medio-largo plazo; se observa cómo la dependencia económica del turismo internacional que presentan determinados destinos turísticos, como Málaga, está motivando el establecimiento de medidas que implican la flexibilización de las normas sanitarias con el fin de salvar la temporada turística; por ejemplo, permitiendo la llegada de turistas en Semana Santa aun con restricciones de movilidad entre provincias (Martínez 2021). Por otro lado, esta situación ha beneficiado a grandes empresas multinacionales que han conseguido multiplicar sus ingresos, principalmente, las vinculadas al capitalismo de plataforma (Glovo y Deliveroo), el entretenimiento en streaming (Neflix, HBO...), así como las grandes compañías de distribución como Amazon (Mansilla 2021).

En el contexto académico, son varios los autores que consideran que la pandemia puede presentarse como una oportunidad para la necesaria transformación de la industria turística y el contexto en el que opera (Everingham \& Chassagne 2020, Hall et al. 2020, Ioannides \& Gyimóthy 2020, Prideaux et al. 2020). Así, encontramos enfoques críticos que proyectan un turismo desvinculado de las demandas del capital que podría funcionar como "opción de relación social y cultural, que ayude a la realización de una vida plena y una 
desaceleración comunitaria creativa" (Acosta 2021: 113). Surgen también diversas reflexiones y propuestas desde enfoques como el decrecimiento (Fletcher et al. 2021), el post-crecimiento (Romero-Padilla et al. 2020) o el ecologismo (Higgins-Desbiolles 2020, Ramis 2021). En este sentido, es destacable la carta abierta de la ONG Transforming Tourism Initiative (2020) al secretario general de la OMT, que supone el reconocimiento de la gran oportunidad que representa esta crisis para acometer la transformación que la industria precisa. No obstante, para ello es necesario configurar y poner en marcha la correlación de fuerzas que permita la realización de este futuro Post-Covid-19 (Mansilla 2021).

\section{Metodología}

El carácter de la investigación es fundamentalmente exploratorio al situarse en un contexto nuevo y de alta incertidumbre por los rápidos $\mathrm{y}$ constantes cambios que envuelven la crisis provocada por la Covid-19. El ámbito de estudio es la ciudad de Málaga, donde se trabaja con tres casos de conflicto en el espacio turístico. Se parte de las investigaciones previas de Navarro-Jurado et al. (2019) y Romero-Padilla et al. (2019) que analizan estos casos de conflicto en la ciudad de Málaga en una situación de pre-pandemia, lo que permite contrastar si en la situación actual de crisis se refuerzan las percepciones o se producen cambios. Se sigue una metodología similar en lo que respecta al empleo de métodos cualitativos para explorar y analizar la percepción de los movimientos sociales sobre los conflictos estudiados. Los métodos cualitativos van ganando peso en las investigaciones sobre los espacios turísticos y sus actores, pues permiten una mayor aproximación a la realidad de los hechos que acontecen en el mundo social (Beard et al. 2016, Huete et al. 2008).

En concreto, se han utilizado, por un lado, técnicas de observación e investigación documental. Fundamentalmente se han revisado y sistematizado las investigaciones previas sobre los casos estudiados en un contexto de pre-pandemia, documentos publicados durante la pandemia y revisión selectiva de prensa. Por otro lado, se han realizado entrevistas semiestructuradas a los movimientos sociales en la actual situación de pandemia. Los temas que han guiado estas entrevistas han sido la percepción sobre el modelo turístico de Málaga; el estado en que se encuentra el objeto del conflicto y el propio movimiento; los 
cambios percibidos debido a la pandemia; y reflexiones sobre las perspectivas de futuro tras la crisis sanitaria. Para analizar esto, las entrevistas se centran en sus percepciones en relación con la situación previa a la aparición de la Covid-19, la situación presente y las perspectivas de futuro y/o posibilidades de cambio. Estas entrevistas constituyen la fuente de información principal en torno a la que se estructuran los resultados de este artículo. En la tabla II se muestra el detalle de las herramientas metodológicas utilizadas.

\section{Tabla III. Herramientas metodológicas.}

\begin{tabular}{|c|c|}
\hline Herramientas & Temas de trabajo \\
\hline $\begin{array}{l}\text { Observación } \\
\text { e investigación } \\
\text { documental } \\
\text { (abril 2020 - } \\
\text { abril 2021) }\end{array}$ & $\begin{array}{l}\text { Identificación de casos de estudio. Se establece como requisito dis- } \\
\text { poner de información primaria recabada en un contexto pre-Covid } \\
\text { sobre los movimientos sociales analizados y los conflictos en los que } \\
\text { se encuentran involucrados. } \\
\text { Análisis de información sobre efectos de la crisis sanitaria y las res- } \\
\text { tricciones sobre el turismo en el ámbito de Málaga. } \\
\text { Sistematización de la información y desarrollo de fichas de análisis de } \\
\text { los conflictos y los movimientos sociales analizados. }\end{array}$ \\
\hline $\begin{array}{l}\text { Entrevistas } \\
\text { (enero - marzo } \\
2021 \text { ) } \\
7 \text { participantes: } 7 \\
\text { hombres }\end{array}$ & $\begin{array}{l}\text { Revisión de las fichas de análisis elaboradas sobre el conflicto. } \\
\text { Temáticas de las entrevistas: } \\
\text { Percepción sobre el modelo turístico de Málaga. } \\
\text { El estado en que se encuentra el objeto del conflicto y el propio } \\
\text { movimiento. } \\
\text { Cambios percibidos debido a la pandemia. } \\
\text { Reflexiones sobre perspectivas de futuro tras la crisis sanitaria. }\end{array}$ \\
\hline
\end{tabular}

Fuente: Elaboración propia

\section{Análisis y resultados}

\section{I Ámbito de Estudio}

Málaga supone un claro ejemplo de crecimiento acelerado y de transformación como ciudad turística que no ha quedado al margen de los conflictos. Se ha afianzado como un destino de atractivo turístico urbano ligado al consumo cultural a nivel global, a través del desarrollo y la puesta en marcha de acciones que la han configurado como la conocida "Ciudad de Museos", presentando en su totalidad 40 museos y centros de interpretación mayoritariamente ubicados en su Centro Histórico (E1 Mundo, 2017). De hecho, la 
mayor parte de la actividad turística se aglutina en este espacio de la ciudad, si bien, las últimas tendencias apuntan a que esta concentración de visitantes en el Casco Histórico podría estar dilatándose hacia otras proximidades (López 2020, Romero-Padilla et al. 2019)

Asimismo, la ciudad de Málaga ha protagonizado una profunda evolución y rehabilitación urbana planificada en su Casco Antiguo que ha contribuido a lo largo de estos años al establecimiento de nuevas actividades comerciales y económicas que se han visto beneficiadas por el incremento de la llegada de turistas (Marin-Cots et al. 2017). Pese a esto, dichos cambios han contribuido a un descontento generalizado de la población residencial, debido a la consideración de que no existe un equilibrio entre las nuevas actividades y las tradicionales, o entre la creciente densidad de turistas en comparación con la de residentes. Estos datos se han traducido en la reducción de la población residente en el centro de la ciudad, la cual ha descendido paulatinamente desde la década de los 90, así en 1991 se registraron 6868 residentes, que pasaron a ser 4952 en el año 2015 (OMAU 2020, Ayuntamiento de Málaga 2015). En 2020, los datos seguían mostrando esta tendencia alarmista a la baja, ya que solo se encontraban empadronadas en este distrito 4300 personas, aunque para más énfasis en el asunto, la Asociación de Vecinos Centro Antiguo de Málaga estimó que la totalidad de residentes no superaba los 3000 (Sánchez 2020).

Esto demuestra el gran contraste existente entre las cifras de la población residencial y las de los cinco millones de visitantes que recibe anualmente la ciudad. En este sentido, según datos del registro de turismo de la Junta de Andalucía, en 2018 el Centro Histórico de Málaga tenía la mayor concentración de plazas en viviendas con fines turísticos con 2870, un número que supone más del doble de la cifra del siguiente barrio con más plazas (OMAU 2020).

Por tanto, los impactos negativos de la mayor presión turística en zonas residenciales derivan como consecuencia del desarrollo de un modelo turístico sin límites. De esta forma, el número de llegadas de visitantes ha seguido un incremento paulatino anual, cerrando el año 2019 con las mayores cifras desde que se registran, superando los 1,4 millones de turistas hospedados en sus alojamientos hoteleros y excediendo las 2,7 millones de pernoctaciones, convirtiéndose así en el segundo destino urbano español con mayor grado de 
ocupación en sus hoteles, con una media del 78,6\% (Diario Sur 2020). Concretamente, la provincia superó por primera vez los 13 millones de viajeros en este mismo año, lo que trajo consigo un impacto mayor a los 14000 millones de euros. Estas cifras suponían un aumento de un 4,2\% de los visitantes que llegaban a Málaga respecto al año anterior (La Opinión de Málaga 2019).

El turismo se convierte así en el sector productivo de mayor importancia para el territorio, transformándolo por completo, como también a su sociedad y a su economía. Sin embargo, la apuesta por el crecimiento turístico que ha protagonizado la ciudad de Málaga ha traído consigo la aparición de impactos negativos que han sido más notables conforme han ido creciendo las cifras del sector dando lugar a conflictos en este espacio turístico. A partir de las investigaciones de Navarro-Jurado et al. (2019) y Romero-Padilla et al. (2019), se pueden identificar tres casos de conflicto en la ciudad de Málaga motivados por el crecimiento turístico a través de proyectos inmobiliario-turísticos y del incremento de la capacidad de alojamiento. Estos tres casos son los que se utilizan en este estudio para analizar las percepciones que tienen los movimientos sociales involucrados en este contexto de pandemia:

- Hotel-Rascacielos en el puerto de Málaga. Proyecto respaldado por un grupo inversor catarí sin experiencia en el sector hotelero. Supone la construcción de un rascacielos de 27 plantas y 116 metros de altura ( sin contar el basamento) en terrenos públicos portuarios (Sánchez 2021), lo cual deriva en la transformación de la zona y la incorporación del uso hotelero en el marco normativo que la regula. En oposición se constituye la Plataforma Defendamos Nuestro Horizonte, con el apoyo de aproximadamente 30 instituciones y comunidades sociales.

- Torres Repsol. Este proyecto pretende la reconversión de una zona industrial con la construcción de cuatro rascacielos dirigidos a viviendas y oficinas, uso hotelero y zonas comerciales, equipamiento de dominio público, cuatro torres para 400 VPO y un parque de 65000 metros cuadrados en unos terrenos de 177548 metros cuadrados (Jiménez 2020). Debido a su ubicación, se pretende promocionar como una "nueva centralidad urbana" (Ayuntamiento de Málaga 2011) por su proximidad al Museo Ruso, al Museo del automóvil y al paseo marítimo. Surge en 2016 la Plataforma Ciudadana Bosque Urbano Málaga, pro- 
poniendo como alternativa convertir los terrenos en una gran área de bosque urbano con especies vegetales autóctonas mediterráneas. $\mathrm{Su}$ propuesta parte del uso como zona verde que estaba establecido en los PGOU anteriores.

- Centro Histórico de Málaga. En este caso el conflicto es derivado de los procesos de turistificación y gentrificación acelerados. El principal problema que se percibe es la convivencia entre la población local residente y los turistas ante una situación de crecimiento exponencial de la oferta turística, especialmente la de alojamiento. Este caso es de vital importancia, ya que el desarrollo del turismo está tan presente en el espacio que cualquier planificación integra esta actividad como centro de cualquier proyección (Navarro et al. 2019). La Asociación de Vecinos Centro Antiguo de Málaga se constituyó en 1992, aunque en los últimos años ha comenzado a actuar con mayor visibilidad y presencia en los medios ante la insostenibilidad de los conflictos.

Sin embargo, la llegada de la crisis pandémica ha supuesto la paralización de la economía malagueña, siendo las actividades relacionadas con el turismo las más perjudicadas en consonancia con las tendencias nacionales. Según el Balance Turístico de la Costa del Sol para 2020 (Turismo y Planificación Costa del Sol 2021) la provincia de Málaga experimentó un descenso respecto al año anterior en las llegadas de pasajeros por Aeropuerto, AVE y Cruceros del $-74,1 \%,-61,1 \%$ y $-89,3 \%$, respectivamente. El mismo balance muestra que el número de empresas y empleos turísticos según afiliaciones a la Seguridad Social disminuyó en un $-13,4 \%$ y un $-14,7 \%$, respectivamente. Por su parte, con base en los datos de la Encuesta de Ocupación Hotelera (INE 20192020) para la ciudad de Málaga durante los 8 meses en los cuales no hubo confinamiento domiciliario, se obtiene que en 2020 hubo un descenso de un $-28,12 \%$ en el número de establecimientos hoteleros abiertos, un grado de ocupación hotelera un 51,56\% menor y una disminución del $-32,67 \%$ en el personal empleado en este tipo de establecimientos.

En relación con la planificación estratégica, el Ayuntamiento de Málaga ha presentado un Plan de Reactivación para la ciudad de Málaga enfocado en la dinamización de la ciudad y la transformación de su tejido productivo con proyectos orientados a la obtención de financiación procedente de los 
fondos Next Generation EU (Ayuntamiento de Málaga 2020). Por un lado, se observan medidas que dan continuidad y fomentan dinámicas conflictivas existentes en el modelo de desarrollo turístico anterior a la pandemia, concretamente en lo referente a propuestas de apoyo al comercio, hostelería y restauración. Ejemplos de estas medidas son la liberalización/habilitación de suelo para las terrazas, agilización de licencias, la liberalización de horarios comerciales o la bonificación de la tasa de terrazas para incentivar la restauración en exteriores. Por otro lado, también se plantean una serie de medidas más alineadas con los objetivos del Pacto Verde Europeo como la creación de un Centro de Inteligencia Turística y Turismo Sostenible, la generación de energía renovable o la apuesta por la economía circular.

\subsection{Los conflictos en el espacio turístico durante la pandemia: percepción de los movimientos sociales}

En relación con el análisis de las percepciones de los movimientos sociales, estos perciben que la aparición y propagación de la pandemia ha legitimado el discurso y reivindicaciones que venían realizando como resistencia al modelo turístico de la ciudad. Esta legitimación del discurso es percibida en la medida en que la Covid-19 ha contribuido a visibilizar la importancia de las cuestiones medioambientales y la calidad de vida, el uso del espacio público común o el riesgo que supone la ausencia de una verdadera participación ciudadana basada en derechos y legislaciones sólidas. Sin embargo, su percepción es que las autoridades locales no apuestan por un cambio en el modelo:

"[...] con la pandemia actual debería haber cambiado todo, puesto que lo que nos enseña esta pandemia es que Málaga y todos en general necesitamos de espacios y de zonas verdes, de espacios públicos más amplios [...] con contacto directo con la naturaleza [...] cada vez hay más evidencias científicas que nos dicen que precisamente el deterioro de la biodiversidad es lo que está generando este tipo de enfermedades [...] Ni una cosa, ni la otra está siendo entendido por los gobernantes del Ayuntamiento de Málaga". (Entrevistado 1)

"[...] el COVID-19 ha venido a levantar la realidad de lo que ya había. Lo que ha venido es a decir: esto es lo que hay y lo que hay es insostenible [...] ¿Qué ocurre? Que muchos nos tememos que el modelo actual, absolutamente negativo reconocido por mucha gente, va a seguir siendo potenciado con 
el dinero público. Ojo, con el dinero de todos. Porque no queremos cambiar, hay muchos intereses metidos ahí". (Entrevistado 2)

De acuerdo con esto, se percibe riesgo de vulneración de derechos bajo la justificación de la necesidad de paliar la crisis que experimentan los sectores vinculados al ocio y al turismo. Esto se observa en la relajación de las obligaciones que deben cumplir las empresas de estos sectores. Un ejemplo es la normativa relacionada con la Declaración de Zonas Acústicamente Saturadas en el distrito Centro de Málaga, que entró en vigor en enero de 2020 (BOP 2020):

"[...] de las indicaciones que plantea el ZAS es que no se pueden abrir nuevos locales de hostelería en zona ZAS y si cierra una tienda de trajes de novia, no puede abrir un bar. Esto, si os dais un paseo por el centro, se está incumpliendo sistemáticamente, pero por decenas de locales. El COVID-19 se está utilizando para potenciar mucho más el modelo extractivo que había antes. O sea, lo que nos viene ahora va a ser muchísimo peor aún”. (Entrevistado 3)

Desde una perspectiva interna, los movimientos sociales perciben las medidas relacionadas con el distanciamiento social como uno de los factores que más ha afectado a sus dinámicas organizativas. Esto ha motivado la pérdida de continuidad, imposibilidad de reuniones, manifestaciones y cualquier tipo de movilización en el espacio público, lo cual es importante para que los movimientos sociales puedan visibilizar sus reivindicaciones:

"A lo que corresponde al funcionamiento de nuestro colectivo, yo echo en falta un cierto mantenimiento de las reuniones que teníamos con anterioridad, más participativas, con compañeros comprometidos con el colectivo [...] también la imposibilidad de poder plantear cualquier movilización que hubiera sido recurrente". (Entrevistado 4)

"Yo creo que esto (situación derivada de la pandemia) ha frenado no solo al Bosque Urbano Málaga, sino a todos los movimientos sociales". (Entrevistado 1)

Se percibe con cierto alivio, que la Covid-19 ha ralentizado los trámites administrativos, lo que ha conseguido paralizar la puesta en marcha de proyectos que, en otras circunstancias, salen adelante con mayor celeridad: 
"En alguna medida, la tramitación que ha llevado a cabo la Gerencia Municipal de Urbanismo de la mano de la Autoridad Portuaria ha tenido una cierta ralentización por las circunstancias de dificultad para trabajar [...] por temas de teletrabajo y demás". (Entrevistado 4)

Los factores anteriormente citados (distanciamiento social y ralentización de trámites administrativos) también han motivado la reflexión interna y reanudación de otras tareas que habían quedado paralizadas de estos movimientos. De esta forma, destacan los saltos de escala que han experimentado las reivindicaciones de algunos de ellos: por un lado, nos encontramos con un salto de escala en los medios de comunicación y en apoyos ciudadanos y, por otro, con un salto de escala de carácter político vinculado a la Agenda 2030 y a la reciente apuesta de determinadas instituciones supralocales por basar la recuperación de esta crisis en una verdadera transición ecológica:

"Lo de las firmas era una asignatura pendiente que teníamos. [...] Primero, salir del ámbito de la provincia, que no lo habíamos conseguido, salvo en alguna situación puntual, y dos, incorporar a personas, como dicen, de 'reconocido prestigio’ más allá del ámbito de Málaga”. (Entrevistado 4)

"[...] al final esto son decisiones políticas [...] por eso queremos seguir hablando con la Dirección General de Políticas Palanca, y con la Secretaría de Estado Agenda 2030". (Entrevistado 1)

Por su parte, uno de los entrevistados nos comenta cómo la pandemia ha hecho trascender a toda la sociedad problemas que anteriormente sufrían sólo los habitantes del centro histórico de Málaga:

"[...] ahora resulta que el problema no es nuestro solo, sino que es un problema de toda la sociedad y de todos los malagueños. Es decir, si hay gente liándola en las calles sin mascarillas va a haber una cuarta ola, otra vez más problemas, va a haber otro confinamiento, la gente no se va a poder mover y demás". (Entrevistado 5)

Desde una dimensión económica y turística, la situación actual de incertidumbre y la forma en que ha afectado al sector turístico ha hecho aumentar las dudas respecto a la viabilidad económica de algunos proyectos que utilizaban el turismo como justificante y ha puesto en evidencia la inexistencia de 
desarrollo turístico sostenible y resiliente; así como la naturaleza especulativa del actual modelo. De esta forma, reconocen la fragilidad del actual modelo turístico y la persistencia por parte de los promotores de estos proyectos por continuar con las dinámicas de acumulación de capital presentes en el contexto pre-pandemia:

"Los análisis que se hacen respecto a cuál va a ser el futuro del proyecto y el análisis de los datos que acompañan la justificación, la necesidad, de este proyecto, están totalmente fuera de juego. Ya estamos hablando de unas proyecciones de un hotel como las que había anteriormente sin, obviamente, unas previsiones hoteleras, una fiabilidad [...]." (Entrevistado 4)

“[...] es un modelo que provoca el éxodo vecinal, pero que encima tiene muchísimas debilidades porque pasa una pandemia o viene cualquier cambio de ciclo de flujos turísticos y se derrumba, como ha pasado...". (Entrevistado 6)

En esta línea, los movimientos sociales recalcan que para que la ciudad y la propia actividad turística tengan un futuro viable el actual modelo requiere una transformación:

"El turismo en Málaga debería funcionar como una forma de relacionarnos con el resto del mundo y ser ciudad; pero hay que tener un modelo, más allá de un eslogan o un branding city ¿no? Un City Branding como los de: Capital Cultural". (Entrevistado 7)

"Se tendría que empezar por la calidad de los Museos. Tenemos unos atractores válidos para un turismo de crucero, a lo sumo, o para el turismo de borrachera $[\ldots]$ son excusas para montar un relato porque si no, estaríamos haciendo cultura de base y estaría funcionando... y el modelo no sería Barcelona, sino que sería Berlín [...] donde quieren seguir manteniendo una identidad de ciudad cultural de verdad". (Entrevistado 3)

"El Bosque Urbano puede ser un espacio donde se puedan hacer actividades culturales $[\ldots]$ que puedan atraer gente en pequeños grupos $[\ldots]$ nos puede atraer a este tipo de visitantes que se preocupe y le guste la naturaleza y quiera verla en vivo, pero también a ese estudiante de primaria, de secundaria, etc". (Entrevistado 1) 
Tras el análisis de las entrevistas a los movimientos sociales se percibe una continuidad e, incluso, intensificación de las dinámicas presentes en el contexto pre-pandemia debido a la inercia del desarrollo turístico-inmobiliario. No obstante, la aparición de la Covid-19 ha puesto de manifiesto las debilidades de dicho modelo y, por tanto, la necesidad de un cambio. En este sentido, a nivel supralocal aparecen oportunidades para la transformación del sector, lo que se suma a una mayor conciencia de los problemas socioeconómicos y medioambientales por parte del conjunto de la sociedad.

\subsection{Discusión de resultados}

Tras la reestructuración económica postfordista y la apuesta por la terciarización de las ciudades consolidadas, el crecimiento turístico-inmobiliario ha ido ganando peso como modelo de acumulación y reproducción del capital (Cañada et al. 2021, Harvey 2013) hasta convertirse en una de las principales y más dinámicas fuerzas productoras del espacio (Lefebvre 1974). Este crecimiento turístico-inmobiliario fue responsable de gran cantidad de impactos negativos en el contexto pre-pandemia y no estuvo exento de críticas por parte de aquellos que sólo percibían los perjuicios y la violencia del crecimiento turístico sin límites (Büscher \& Fletcher 2017, Milano 2018). De esta forma, aparecieron movimientos sociales de resistencia a un modelo de desarrollo turístico que no parecía responder a sus necesidades e intereses, sino, más bien, a los de aquellos que sólo querían convertir sus ciudades en un negocio rentable (Blanco-Romero et al. 2019, Logan \& Molotch 1987).

En este contexto, surgieron estudios que analizaron el choque de intereses existente entre las reivindicaciones de estos movimientos sociales, que luchaban por el derecho a la ciudad (Lefebvre 1968) y la justicia espacial (Soja 2008) en sus espacios cotidianos; y el discurso de una "clase dominante" que apoyaba este crecimiento turístico-inmobiliario y se esforzaba por deslegitimar las reivindicaciones de estos movimientos (Blanco-Romero et al. 2019, Milano 2018). En este sentido, Málaga supone un claro ejemplo de crecimiento acelerado y transformación como ciudad turística que ha ido experimentado estos conflictos conforme aumentaban las cifras turísticas (Navarro-Jurado et al. 2019, Romero-Padilla et al. 2019). No obstante, este contexto ha sufrido un importante cambio con la llegada de la crisis sanitaria 
y económica provocada por la Covid-19, que ha demostrado la debilidad de la industria turística y su modelo de crecimiento, dejando indefensos a los destinos que más dependían de los ingresos del turismo (Mansilla 2021).

Con base en el estudio realizado, a nivel local se percibe que la inercia del crecimiento turístico-inmobiliario sigue orientando las decisiones estratégicas de la ciudad de Málaga pese a una cada vez mayor oposición al modelo. Estos elementos son recurrentes en los estudios de los conflictos en la ciudad y en el entorno turístico (Navarro-Jurado et al. 2019, Romero-Padilla et al. 2019, Blanco-Romero 2019, Milano 2018). En este sentido, destaca la actuación de la Plataforma Defendamos Nuestro Horizonte de enero de 2021 cuando lanza un nuevo manifiesto en contra de la construcción de la Torre del Puerto que fue firmado por 300 personalidades de reconocido prestigio de toda España (Vázquez 2021). Este salto de escala en cuanto a apoyos al movimiento vino acompañado de la presentación de una moción en la que se planteó en el pleno del Ayuntamiento la realización de una consulta ciudadana donde se decidiera de forma democrática si Málaga quería que se construyera esa torre. No obstante, la propuesta fue rechazada en el pleno.

Por otra parte, la aparición y el comportamiento de la pandemia ha reforzado las reivindicaciones de los movimientos sociales en relación con la necesidad de fomentar un desarrollo turístico que priorice las cuestiones sociales y medioambientales (Lefebvre 1968, Soja 2008). Por un lado, el surgimiento de la pandemia viene a demostrar la insostenibilidad del modelo turístico de crecimiento ilimitado en la medida en que la extrema dependencia y especialización turística de los destinos ha limitado su capacidad de respuesta y adaptación en este escenario de incertidumbre (Romero-Padilla et al. 2020). Por otro lado, la actual crisis sanitaria ha motivado la reflexión sobre las posibles conexiones causales entre el surgimiento y la propagación de la Covid-19, la crisis climática y las repercusiones medioambientales de las dinámicas del sector turístico en términos de calentamiento atmosférico, contaminación y agotamiento de recursos (Fletcher et al. 2021, Valdivielso y Adrover 2021).

Finalmente, el ejemplo del salto de escala experimentado por la plataforma Bosque Urbano de Málaga en relación con la Agenda 2030 apunta que, si existen perspectivas de cambio, éstas se ubican en una escala superior 
a la local, ya que la máquina urbana de crecimiento va a seguir presionando por la obtención de ganancias y la intensificación y reproducción de las dinámicas que lo permiten (Alexandri \& Janoschka 2020, Harvey 2013, Logan \& Molotch 1987). Estas oportunidades de cambio que se vislumbran en un nivel supralocal están relacionadas con la reciente apuesta y compromiso por parte de organismos como la Unión Europea o la ONU por una recuperación basada en una transición ecológica y resiliente que, en el caso de Europa, seguiría los principios del Pacto Verde Europeo (Parlamento Europeo 2020). No obstante, la materialización de este deseado cambio en la industria necesita la configuración de la correlación de fuerzas que lo permita (Mansilla 2021). En este sentido, resulta de vital importancia la trascendencia social que han experimentado algunas de las reivindicaciones de los movimientos sociales, así como la unión de fuerzas entre los propios movimientos que, aun con sus particularidades, comparten reivindicaciones fundamentales en relación con el derecho a la vida urbana, los derechos básicos en materia de bienestar y la participación en cuestiones trascendentales para el futuro de sus ciudades. Para ello sería muy útil la actuación conjunta, solidaria y el aprovechamiento de sinergias.

\section{Conclusiones}

Los espacios urbanos han experimentado un considerable crecimiento turístico en las últimas décadas, particularmente tras la crisis económica de 2008, momento a partir del cual comenzaron a intensificarse las voces críticas. Como se ha mostrado en la revisión conceptual, surgieron movimientos sociales de resistencia que agruparon a aquellas personas que percibían los efectos negativos del modelo turístico imperante. En este sentido, la crisis provocada por la Covid-19 no ha hecho sino visibilizar la fragilidad y conflictos presentes en estos destinos. El presente artículo se ha planteado dar respuesta a la pregunta de si la Covid-19 ha supuesto un cambio en la percepción de los movimientos sociales sobre los conflictos que han venido desarrollándose en los espacios turísticos a partir del empleo de un enfoque cualitativo.

Entre los resultados más relevantes de la investigación, destaca cómo en el actual contexto las fuerzas locales que presionan por el valor de cambio (Lefevbre 1974, Logan \& Molotch 1987) siguen persistiendo y reforzando 
las dinámicas inherentes al modelo turístico-inmobiliario pre-pandemia, incluso de forma más agresiva (Alexandri \& Janoschka, 2020). No obstante, la Covid-19 ha puesto en tela de juicio la propia viabilidad económica del modelo demostrando que tiene serias debilidades, lo que ha conseguido que aumenten los apoyos en contra de este tipo de crecimiento turístico. En este sentido, a un nivel supralocal se observan propuestas a medio-largo plazo que pueden conducir a cambios en elementos estructurales del modelo de desarrollo turístico; en tanto que, a nivel local, salvo algunas excepciones, se siguen observando medidas cortoplacistas y dinámicas vinculadas con los intereses de las máquinas de crecimiento urbano-turístico--

Cabe destacar en el presente estudio el contexto de incertidumbre en el que se inscribe. De esta forma, se esboza un escenario en el que se produzca una transformación de las actividades turísticas acorde con la creciente cantidad de factores que nos alertan sobre la insostenibilidad del presente modelo, pero también uno en el que todo vuelva a la situación anterior o en el que incluso se intensifiquen las dinámicas extractivas presentes en el modelo pre-pandemia.

Entre las limitaciones de esta investigación se debe tener en cuenta que se trata de un estudio exploratorio, por lo que la metodología empleada podría reforzarse con otras técnicas de tipo cuantitativo, así como incluir a otros sectores de la población. Esto permitiría contrastar resultados y comprobar si se trata de percepciones generalizadas o corresponden a visiones particulares de estos movimientos sociales.

En cuanto a las líneas futuras de investigación sería interesante replicar el estudio en otros destinos turísticos para comparar cómo ha sido la evolución de los conflictos en otras zonas, así como analizar las percepciones de otro tipo de actores (residentes, empresarios, políticos). Finalmente, dada la incertidumbre del contexto en el que se realiza esta investigación, puede ser de interés trabajar en la proyección de escenarios futuros, teniendo en cuenta no sólo la evolución de la crisis sanitaria sino también de la emergencia climática en los destinos turísticos. 


\section{Referencias}

Acosta, A. (2021). El turismo en la senda del postdesarrollo. [Por ahora en la trampa del COVID-19]. En E. Cañada \& I. Murray (Eds.), \#Tourismpostcovid19 Turistificación confinada (pp. 108-114). Barcelona: Alba Sud.

Aledo, A., Ortiz, G., Aznar, P., Mañas, J. J., Jimeno, I., \& Climent-Gil, E. (2021). Vulnerabilidad social y el modelo turístico-residencial español: escenarios frente a la crisis de la COVID-19. En E. Cañada \& I. Murray (Eds.), \#Tourismpostcovid19 Turistificación confinada (pp. 98-107). Barcelona: Alba Sud.

Alexandri, G., \& Janoschka, M. (2020). 'Post-pandemic' transnational gentrifications: A critical outlook. Urban Studies, 57(15), 3202-3214. https://doi.org/10.1177/0042098020946453

Ayuntamiento de Málaga (2011). Plan General de Ordenación Urbana. Gerencia Municipal de Urbanismo. Recuperado de www.urbanismo. malaga.eu

Ayuntamiento de Málaga (2015). Estrategia Urbana Integrada Sostenible "Perchel Lagunillas" Servicio de Programas Europeos-OMAU Málaga 2015. http://static.omau-malaga.com/omau/subidas/archivos/3/1/7713/edusi-malaga-v2.pdf

Ayuntamiento De Málaga (2020). Plan de Reactivación. Ejes y Proyectos Estratégicos. https://www.malaga.eu/recursos/ayto/prensa/Plan-de-Reactivaci\%C3\%B3n-Ejes-y-Proyectos-Estrat\%C3\%A9gicos.pdf

Beard, L., Scarles, C., \& Tribe, J. (2016). Mess and method: Using ANT in tourism research. Annals of Tourism Research, 60, 97-110. https://doi. org/10.1016/j.annals.2016.06.005

Blanco-Romero, A., Blázquez-Salom, M., Morell, M., \& Fletcher, R. (2019). Not tourism-phobia but urban-philia: Understanding stakeholders' perceptions of urban touristification. Boletín de la Asociación de Geógrafos Españoles, 83, 1-30. https://doi.org/10.21138/bage.2834

Boletín Oficial De La Provincia De Málaga (2020). Boletín Oficial de la Provincia de Málaga. Martes, 21 de enero de 2020. https://www.bopmalaga.es/cve.php?cve=20200121-00229-2020 
Bugalski, Ł. (2020). The undisrupted growth of the airbnb phenomenon between 2014-2020. The touristification of european cities before the covid-19 outbreak. Sustainability (Switzerland), 12(23), 1-20. https:// doi.org/10.3390/su12239841

Büscher, B., \& Fletcher, R. (2017). Destructive creation: capital accumulation and the structural violence of tourism. Journal of Sustainable Tourism, 25(5), 651-667. https://doi.org/10.1080/09669582.2016.11 59214

Cañada, E. \& Murray, E. I. (2021). \#TourismPostCOVID19 Turistificación confinada. Barcelona, España: Alba Sud. http://www.albasud.org/ publ/docs/98.pdf

Capocchi, A., Vallone, C., Pierotti, M., \& Amaduzzi, A. (2019). Overtourism: A literature review to assess implications and future perspectives. Sustainability (Switzerland), 11(12). https://doi.org/10.3390/su10023303

Cocola-Gant, A. (2021). Apartamentos turísticos, COVID-19 y capitalismo de plataformas. En E. Cañada \& I. Murray (Eds.), \#Tourismpostcovid19 Turistificación confinada (pp. 150-154). Barcelona, España: Alba Sud.

Comisión Europea (2020). Plan de recuperación para Europa. Recuperado de https://ec.europa.eu/info/strategy/recovery-plan-europe_es

De La Calle Vaquero, M. (2019). Turistificación de centros urbanos: clarificando el debate. Boletín de La Asociación de Geógrafos Españoles, 83, 1-40. https://doi.org/10.21138/bage.2829

Del Romero, L. (2018). Touristification, Sharing Economies and the New Geography or Urban Conflicts. Urban Science, 2, 104, 1-17. https:// doi:10.3390/urbansci2040104

Diario Sur (2 de enero de 2020). «Málaga cierra el año 2019 con su mejor cifra turística desde que hay registros». Diario Sur. Recuperado de https:// www.diariosur.es/turismo/malaga-cierra-2019-20200102170452-nt. html\#: :text=La\%20ciudad\%20de\%20M\%C3\%A1laga\%20ha,ocupaci\%C3\%B3n\%20del\%2079\%20por\%20ciento

Parlamento Europeo (5 de mayo de 2020). «COVID-19: el plan de recuperación de la UE debe priorizar el clima». Noticias Parlamento Europeo. Recuperado de https://www.europarl.europa.eu/news/es/headlines/ society/20200429STO78172/covid-19-el-plan-de-recuperacion-dela-ue-debe-priorizar-el-clima 
Everingham, P., \& Chassagne, N. (2020). Post COVID-19 ecological and social reset: moving away from capitalist growth models towards tourism as Buen Vivir. Tourism Geographies, 22(3), 555-566. https://doi. org/10.1080/14616688.2020.1762119

Fletcher, R., Murray, I., Blázquez, M., \& Blanco, A. (2021). Turismo, decrecimiento y la crisis del COVID-19. En E. Cañada \& I. Murray (Eds.), \#Tourismpostcovid19 Turistificación confinada (pp. 259-263). Barcelona, España: Alba Sud.

Fletcher, R., Murray Mas, I., Blanco-Romero, A., \& Blázquez-Salom, M. (2019). Tourism and degrowth: an emerging agenda for research and praxis. Journal of Sustainable Tourism, 27(12), 1745-1763. https:// doi.org/10.1080/09669582.2019.1679822

García-Hernández, J. S., Armas-Díaz, A., \& Del Carmen Díaz-Rodríguez, M. (2020). Home dispossession and touristification in Santa Cruz de Tenerife (Canary Islands-Spain): Tenant evictions in the neighborhood of El Toscal. Boletín de La Asociación de Geógrafos Españoles, 87. https://doi.org/10.21138/BAGE.2982

Gössling, S., Scott, D., \& Hall, C. M. (2020). Pandemics, tourism and global change: a rapid assessment of COVID-19. Journal of Sustainable Tourism, 1-20. https://doi.org/10.1080/09669582.2020.1758708

Hall, C. M., Scott, D., \& Gössling, S. (2020). Pandemics, transformations and tourism: be careful what you wish for. Tourism Geographies, 22(3), 577-598. https://doi.org/10.1080/14616688.2020.1759131

Harvey, D. (2013). Ciudades rebeldes. Del derecho a la ciudad a la revolución urbana. Madrid, España: Akal.

Hiernaux, D., \& González, C. I. (2014). Turismo y gentrificación: pistas teóricas sobre una articulación. Revista de Geografía Norte Grande, 58, 55-70. http://dx.doi.org/10.4067/S0718-34022014000200004

Higgins-Desbiolles, F. (2020). Socialising tourism for social and ecological justice after COVID-19. Tourism Geographies, 22(3), 610-623. https://doi.org/10.1080/14616688.2020.1757748

Hosteltur (3 DE FEBRERO DE 2021). «España recibió 19 M de turistas en 2020, la mayor caída de su historia». Hosteltur. Recuperado de https://www.hosteltur.com/142043_espana-recibio-19-m-de-turistasen-2020-el-peor-dato-de-su-historia.html 
Huete, R., Mantecón, A., \& Mazón, T. (2008). De qué hablamos cuando hablamos de turismo residencial. Cuadernos de Turismo, 22, 101-121. https://revistas.um.es/turismo/article/view/48091/46071

Instituto Nacional de Estadística (2019-2020). Encuesta de ocupación hotelera. Recuperado de www.ine.es

Ioannides, D., \& Gyimóthy, S. (2020). The COVID-19 crisis as an opportunity for escaping the unsustainable global tourism path. Tourism Geographies, 22(3), 624-632. https://doi.org/10.1080/14616688.2020.1 763445

Ioannides, D., Röslmaier, M., \& Van Der Zee, E. (2019). Airbnb as an instigator of 'tourism bubble' expansion in Utrecht's Lombok neighbourhood. Tourism Geographies, 21(5), 822-840. https://doi.org/10.1080/ 14616688.2018.1454505

Jackson, T. (2009). Prosperity without Growth. Economics for a Finite Planet. Earthscan, Londres: Sterling, VA.

Jiménez, F. (20 de agosto de 2020). «Urbanismo abre el plazo de alegaciones para el desarrollo de los terrenos de Repsol». Diario Sur. Recuperado de https://www.diariosur.es/malaga-capital/urbanismo-abre-plazo-20200819171745-nt.html

Jover, J., \& Díaz-Parra, I. (2020). Gentrification, transnational gentrification and touristification in Seville, Spain. Urban Studies, 57(15), 30443059. https://doi.org/10.1177/0042098019857585

Klein, N. (2010). La doctrina del shock. El auge del capitalismo del desastre. Barcelona: Paidós.

La Opinión de Málaga (30 de diciembre de 2019). «La Costa del Sol supera 13 millones de visitantes en 2019». La Opinión de Málaga. Recuperado de https:/www.laopiniondemalaga.es/malaga/2019/12/30/costa-sol-supera-13-millones-27666869.html

Lefebvre, H. (2017). El derecho a la ciudad. Madrid, España: Capitán Swing Libros. [1968]

Lefebvre, H. (2013). La producción del espacio. Madrid, España: Capitán Swing Libros. [1974]

Logan, J. R., \& Molotch, H. L. (1987). Urban Fortunes: The Political Economy of Place. Los Ángeles: University of California Press. 
López Padilla, M. D. (2020). Procesos actuales de turistificación en la ciudad de Málaga. Boletín de la Real Sociedad Geográfica, CLV, 107-140. https://www.boletinrsg.com/index.php/boletinrsg/article/view/104

Mansilla, J. (2021). El futuro de las ciudades turísticas tras la pandemia. En E. Cañada \& I. Murray (Eds.), \#Tourismpostcovid19 Turistificación confinada (pp. 159-163). Barcelona: Alba Sud.

Marín-Cots, P., Guevara-Plaza, A., \& Navarro-Jurado, E. (2017). Renovación urbana y masificación turística en la ciudad antigua: pérdida de población y conflictos sociales. Ciudad y Territorio Estudios Territoriales 193, 49(1), 453-468. https://recyt.fecyt.es/index.php/CyTET/article/ view/76572

Martínez, P. (23 de marzo de 2021). «El alcalde de Málaga defiende la llegada de turistas pese a las restricciones a la movilidad en la provincia». Diario Sur. Recuperado de https://www.diariosur.es/ turismo/alcalde-malaga-defiende-llegada-turistas-restricciones-coronavirus-20210323182232-nt.html

Milano, C. (2018). Overtourism, Social Unrest and Tourismphobia. A controversial debate. PASOS. Revista de Turismo y Patrimonio Cultural, 18(3), 551-564. http://www.pasosonline.org/Publicados/16318/ PS318_01.pdf

Ministerio de Sanidad (2021). Actualización no 351. Enfermedad por el coronavirus (COVID-19). 12.04.2021. https://www.mscbs.gob.es/profesionales/saludPublica/ccayes/alertasActual/nCov/documentos/Actualizacion_223_COVID-19.pdf

Mundo, El (18 de abril de 2017). «Málaga presenta su marca como capital de museos». El Mundo. Recuperado de https://www.elmundo.es/andalucia/2017/04/18/58f63b1446163fbe378b4575.html

Murray, I., \& Cañada, E. (2021). Turistificación confinada. En E. Cañada \& I. Murray (Eds.), \#Tourismpostcovid19 Turistificación confinada (pp. 14-80). Barcelona: Alba Sud.

Navarro-Jurado, E., Romero-Padilla, Y., Romero-Martínez, J. M., Serrano-Muñoz, E., Habegger, S., \& Mora-Esteban, R. (2019). Growth machines and social movements in mature tourist destinations Costa del Sol-Málaga. Journal of Sustainable Tourism, 27(12), 1786-1803. https://doi.org/10.1080/09669582.2019.1677676 
Nofre, J., Giordano, E., Eldridge, A., Martins, J. C., \& Sequera, J. (2018). Tourism, nightlife and planning: challenges and opportunities for community liveability in La Barceloneta. Tourism Geographies, 20(3), 377-396. https://doi.org/10.1080/14616688.2017.1375972

Oklevik, O., Gössling, S., Hall, C. M., Steen Jacobsen, J. K., Grøtte, I. P., \& Mccabe, S. (2019). Overtourism, optimisation, and destination performance indicators: a case study of activities in Fjord Norway. Journal of Sustainable Tourism, 27(12), 1804-1824. https://doi.org/10.1080/0 9669582.2018 .1533020

Observatorio de Medio Ambiente Urbano (2020). Aproximación al turismo en málaga [proyecto Alter Eco de turismo sostenible]. http:// static.omau-malaga.com/omau/subidas/archivos/1/2/8421/aproximacion-al-turismo-en-malaga-\%28alter-eco\%29_v5.pdf

Organización de las Naciones Unidas. (2020). Informe de políticas: La COVID-19 y la transformación del turismo. ONU-UNWTO, 30. https:// www.un.org/sites/un2.un.org/files/policy_brief_covid-19_and_transforming_tourism_spanish.pdf

Perkumienè, D., \& Pranskūnienè, R. (2019). Overtourism: Between the Right to Travel and Residents' Rights. Sustainability, 11(7), 2138. https:// doi.org/10.3390/su11072138

Prideaux, B., Thompson, M., \& Pabel, A. (2020). Lessons from COVID-19 can prepare global tourism for the economic transformation needed to combat climate change. Tourism Geographies, 22(3), 667-678. https://doi.org/10.1080/14616688.2020.1762117

Ramis, M. (2021). Y después de todo esto, ¿qué? In E. Cañada \& I. Murray (Eds.), \#Tourismpostcovid19 Turistificación confinada (pp. 264-272). Barcelona: Alba Sud.

Romero-Padilla, Y., Cerezo-Medina, A., Navarro-Jurado, E., Romero-Martínez, J. M., \& Guevara-Plaza, A. (2019). Conflicts in the tourist city from the perspective of local social movements. Boletín de La Asociación de Geógrafos Españoles, 83, 1-35. https://doi.org/10.21138/ bage. 2837 
Romero-Padilla, Y., Romero-Martínez, J. M., \& Navarro-Jurado, E. (2020). Reflexiones desde el post-crecimiento: ideas, estrategias y tácticas para el turismo post-covid-19. En L. M. Rondón García, L. Troitiño Torralba, \& C. Mulet Forteza (Eds.), Turismo Post COVID-19. El turismo después de la pandemia global. Análisis, perspectivas y vías de recuperación (pp. 57-76). Ediciones Universidad Salamanca. https:// doi.org/https://doi.org/10.14201/0AQ0296

Russo, A. P., \& Scarnato, A. (2018). "Barcelona in common": A new urban regime for the 21 st-century tourist city? Journal of Urban Affairs, 40(4), 455-474. https://doi.org/10.1080/07352166.2017.1373023

Sánchez, N. (8 de mayo de 2020). «Un pueblo en el centro de Málaga». El País. Recuperado de https://elpais.com/espana/2020-05-07/un-pueblo-en-el-centro-de-malaga.html

Sánchez, N. (5 de febrero de 2021). «El vértigo de Málaga a su rascacielos». El País. Recuperado de https://elpais.com/economia/2021-02-04/ el-vertigo-de-malaga-a-su-rascacielos.html

Soja, E. W. (2008). Postmetrópolis. Estudios críticos sobre ciudades y regiones. Madrid: Traficante de Sueños. https://www.traficantes.net/sites/ default/files/pdfs/Postmetr\%C3\%B3polis-TdS.pdf

Trancoso González, A. (2018). Venice: The problem of overtourism and the impact of cruises. Investigaciones Regionales, 42, 35-51. https://investigacionesregionales.org/wp-content/uploads/sites/3/2019/01/03-TRANCOSO.pdf

Transforming Tourism Initiative (2020). Open Letter: COVID 19- now is the time to transform tourism. http://www.transforming-tourism.org/fileadmin/baukaesten/sdg/downloads/Working_Programme/Open_Letter/Transforming_Tourism_Letter_to_UNWTO_01.pdf

Turismo y Planificación Costa del Sol (2021). Balance del año turístico 2020. Recuperado de www.costadelsolmalaga.org

United Nations World Tourism Organization (28 de enero de 2021). «2020: El peor año de la historia del turismo, con mil millones menos de llegadas internacionales». UNWTO. Recuperado de https://www.unwto.org/es/taxonomy/term/347

United Nations Word Tourism Organization (2018). “Overtourism”? - Understanding and Managing Urban Tourism Growth beyond Perceptions. https://www.e-unwto.org/doi/epdf/10.18111/9789284420070 
Valdivielso, J., \& Adrover, J. (2021). Turismocracia: la vulnerabilidad organizada. En E. Cañada \& I. Murray (Eds.), \#Tourismpostcovid19 Turistificación confinada (pp. 164-172). Barcelona: Alba Sud.

Vázquez, A. (14 de enero de 2021). «Más de 300 personalidades firman un manifiesto contra el rascacielos del Puerto». La Opinión de Málaga. Recuperado de https://www.laopiniondemalaga.es/malaga/2021/01/14/300-personalidades-firman-manifiesto-rascacielos-29297567.html 
